\title{
Advances in gender preselection in swine
}

\author{
L. A. Johnson \\ Germplasm and Gamete Physiology Laboratory, Agricultural Research Service, US Department of \\ Agriculture, Beltsville, MD 20705, USA
}

\begin{abstract}
Gender preselection using isolated populations of $\mathrm{X}$ - and Y-chromosome bearing spermatozoa that have been separated on the basis of DNA content is currently possible in swine and other farm animals, as well as in humans. Semen from most livestock species can now be successfully separated into predominantly $X$ or $Y$ sperm populations before their use for intra-tubal insemination, deep-uterine insemination or for in vitro fertilization (IVF) to produce sexed offspring. Birth of progeny of the desired sex in cattle, sheep and swine under semi-practical conditions has successfully validated the sexing technology. Spermatozoa are separated on the basis of inherent differences in DNA content in the X- and Y-chromosome bearing sperm population using modified flow cytometry/cell sorting technology. Spermatozoa are stained with Hoechst 33342 which binds to the DNA in an amount proportional to the amount of DNA present in the individual spermatozoa. Over 300 animals from various species at several locations have been born using the USDA-Beltsville Sperm Sexing Technology for separating $X$ and $Y$ spermatozoa. Sex ratios are shifted from the normal 50:50 to 85 to $90 \%$ of one sex or the other. In swine, offspring have been born as the result of surgical intratubal insemination of separated spermatozoa and also from IVF and embryo transfer. At the present time, standard swine artificial insemination techniques are not optimized for use with the small numbers of flow cytometrically separated $X$ or $Y$ sperm populations. Cattle, swine and rabbit offspring have been reproduced through the second generation with normal morphology and reproductive function. Numerous improvements have been made in the sexing technology since it was first reported in 1989. Increasing the speed of the sexing process to make the application of the technology available to a larger segment of the livestock industry is paramount, even with insemination technology designed for small numbers of spermatozoa.
\end{abstract}

\section{Introduction}

Use of sexed semen for production of livestock of preselected sex is dependent on economics, effectiveness, efficiency and ease of use. The most cost effective approach for achieving sex preselection involves separating the $X$-from the $Y$-chromosome bearing spermatozoa followed by use for artificial insemination (AI) or for in vitro fertilization (IVF) and subsequent embryo transfer (ET). Since one is pre-determining the outcome of conception rather than the outcome after fertilization, the use of X- or $\mathrm{Y}$ - bearing spermatozoa has a clear advantage over embryo sexing. Livestock management schemes can benefit from sex preselection because of the ability to plan matings for a specific sex. In addition to faster genetic progress from sexed spermatozoa or embryos, there are additional advantages of management efficiency and facility flexibility. The swine industry would have a more efficient and economically advantageous means of producing male and female hybrid lines.

Our report (Johnson et al., 1989) of effective sex preselection in rabbits was the first report detailing the use of DNA as a marker for sex preselection and subsequent birth of offspring of the predicted sex. This was followed by publication of results confirming use of the method with boar semen (Johnson, 1991). Since 1989, more than 300 animals have been born using this procedure 
Table 1. Markers that have been used or proposed for separation of $X$ and $Y$ spermatozoa

\begin{tabular}{lll}
\hline Proposed marker & \multicolumn{1}{c}{ Parameter } & \multicolumn{1}{c}{ Reference } \\
\hline DNA & X spermatozoa $>$ Y & Moruzzi, JF., 1979 \\
Size & X spermatozoa $>$ Y & Cui, K., 1997 \\
Motility & Y faster than X & Ericsson et al., 1973 \\
F-Body & Long arm on Y chromosome & Barlow and Vosa 1970 \\
H-Y antigen & Spermatozoa surface & Hendriksen et al., 1993 \\
Protein & Spermatozoa surface & Hendriksen et al., 1996 \\
\hline
\end{tabular}

(Cran et al., 1993,1995; Rath et al., 1997; Seidel et al., 1997; Catt et al., 1996). Many of the animals were produced at other locations where the instrumentation has been duplicated thus confirming the reproducibility of the technology. This review will describe the process involved in using the Beltsville Sperm Sexing Technology with particular emphasis on swine. Current usage as well as future prospects for use in practical situations will be discussed. Since the Beltsville technology is the only sex preselection technology that has been proven effective in livestock to date, it will be the primary emphasis of this review.

\section{Basis for Separation of Spermatozoa}

\section{Physical separation methods}

Numerous attempts to separate $X$ - from Y-chromosome bearing spermatozoa have been tested over the last 70 years (Table 1); most protocols are based on some physical aspect of the spermatozoa. None of the various protocols that use a supposed physical difference between $X$ and $Y$ spermatozoa have proven effective in obtaining a higher proportion of either sex. Our experience in testing these methods (Johnson, 1988) is that no method developed on the basis of an inherent physical difference within the spermatozoa or on the sperm surface has been effective in separating $X$ - from Y-bearing spermatozoa, except a method based on DNA content of the spermatozoa. Overviews of the various methods that have been tried have been published by Kiddy and Hafs (1971), Amann and Seidel (1982), Gledhill (1988) and Johnson (1992, 1994, 1995).

\section{Difference in DNA content for separation of $X$ and $Y$ spermatozon}

It is well established that the DNA content of X-and Y-chromosome bearing spermatozoa is different in most mammals. The $Y$ chromosome is smaller and carries less DNA than the $X$ chromosome, while the autosomes carried by $X$ - or Y-bearing spermatozoa are identical in DNA content. Taking advantage of this difference to separate $X$ - and $Y$-bearing spermatozoa has only been possible since the development of flow cytometry. Initial attempts to differentiate $X$ and $Y$ spermatozoa using DNA and flow cytometric analysis did not succeed (Gledhill et al., 1976). However, with the combination of improved staining methods and a realization that aspherical cells such as spermatozoa must be orientated to the excitation source (Dean et al., 1978), the relative difference in DNA could be measured (Pinkel et al., 1982). These initial advances in analysis of sperm DNA formed the basis for routine analysis of sperm DNA using modified commercial instrumentation (Johnson and Pinkel, 1986). Sorting sperm populations on the basis of difference in DNA followed (Johnson et al., 1987a; Johnson and Clarke, 1988). Improved cellular staining and the use of vital fluorochromes to label DNA led to the separation of X-and Y-chromosome bearing spermatozoa for the production of offspring of pre-determined sex (Johnson et al., 1987b, 1989; Johnson, 1991). 


\section{Separation of Spermatozoa by Flow Cytometry}

The sorting of the spermatozoa is done using a modified version of any full scale flow cytometer/cell sorter. Much of the equipment in use today was designed and built in the early 1980s. Basic flow cytometric system principles have not changed since their introduction in the late 1960s and early 1970s. The adaptation of commercial flow cytometers with modifications for aspherical cells such as spermatozoa (Johnson and Pinkel, 1986) can still be applied to more recent models of flow cytometer/cell sorters (Fig. 1). The older units continue to work as effectively as the newer ones with the exception that the data acquisition system is slower. A flow cytometer/cell sorter system adapted for sperm sorting uses a five watt water cooled argon laser as an excitation source. Although fluorochromes designed to bind to DNA generally give off a bright signal, the five watt water cooled laser remains the laser of choice for most DNA fluorochromes.

\section{Resolving $X$ and $Y$ spermatozoa based on DNA difference}

The resolution of X-and Y-bearing spermatozoa using flow cytometric DNA analysis is difficult compared with that of other types of cell. The inordinate compactness of chromatin in the morphologically flat, paddle or ovoid shaped sperm head that is characteristic for domestic animals causes a high index of refraction. The difference in refractive index between the sperm head and the surrounding medium, coupled with the flat shape of the sperm head, results in preferential emission of light in the plane of the cell (from the edge of the sperm head). The orientation of the sperm head with respect to the excitation laser beam and the optical detectors is therefore critical for resolution. The modification of the flow cytometer/cell sorter system consists of a forward fluorescence detector in place of the light scatter detector that is standard to orthogonal configured flow systems. This is necessary so that fluorescent light from both the edge of the spermatozoa $\left(90^{\circ}\right)$ as well as from the flat side $\left(0^{\circ}\right)$ of the spermatozoa can be collected. The second modification is to replace the usual cylindrical sample input needle with a similar but bevelled sample input needle (Johnson and Pinkel, 1986). The modified flow cytometer/cell sorter is essential for attaining separate populations of $\mathrm{X}$ - and $\mathrm{Y}$-chromosome bearing spermatozoa based on differences in DNA content and for reanalysing sorted spermatozoa to determine the proportions of $X$ or $Y$ spermatozoa in a given sorted sample (Fig. 1; Johnson et al., 1987a, 1989).

\section{Instrumentation options for sexing spermatozoa by flow sorting}

Differentiation of the amount of DNA present in the X-and Y-chromosome bearing spermatozoa for sorting can be done on nearly all commercial flow cytometer/cell sorters that have been manufactured in the past 15-20 years if they have been properly modified. To date flow cytometer/cell sorter systems manufactured by the Coulter Corporation, Hialeah, FL (EPICS V; EPICS 750 series, ELITE) and the Becton Dickinson Co., Mountain View, CA (FACS Vantage; FACStar Plus; FACStar; FACS 440) have been modified for sperm sorting. Recently we have modified a high speed flow cytometer/cell sorter manufactured by Cytomation Inc., Fort Collins, $\mathrm{CO}$ (MoFlo). The high speed MoFlo system has the capability of sorting at a rate four to six times that of the older standard systems (L. Johnson and G. Welch, unpublished). Each of the systems mentioned was modified to provide for a bevelled sample injection needle and a second fluorescence detector located $0^{\circ}$ to the laser (Fig. 1). This modification allows for orientation of the spermatozoa as it passes from the bevelled needle and past the laser beam and for the selection of the orientated population through electronic gating (Johnson and Pinkel, 1986).

\section{Sperm preparation prior to sorting}

Protocols for the preparation of fresh semen for flow sorting have been developed based on the principle that the less insult imposed on the spermatozoa, the greater the likelihood that the ultimate 


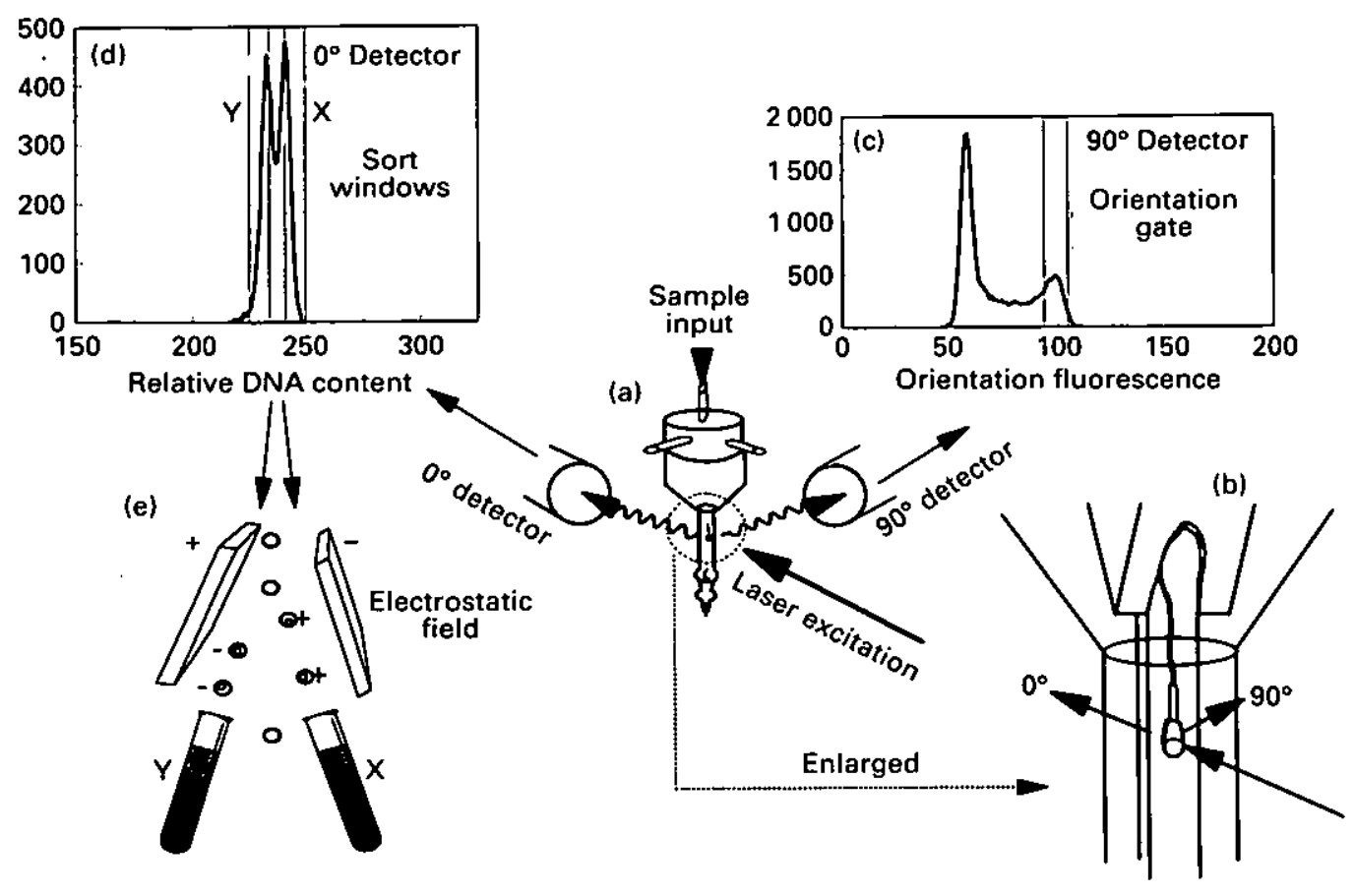

Fig. 1. A diagram of the sample input and data handling capability of a flow cytometer/cell sorter modified for resolution of $X$ - from $Y$-chromosome bearing spermatozoa based on their difference in DNA content according to the Beltsville Spermatozoa Sexing Technology. Spermatozoa enter through a needle labelled 'sample input' and are passed into the flow cell (a). They leave the needle into the flow nozzle in single file and are oriented according to the position of the needle (b) which has been ground to a bevel on the exit end. Orientation occurs when the opposing flatter surfaces of the spermatozoa head simultaneously face the laser and $0^{\circ}$ forward detector, with the edge directed to the $90^{\circ} \mathrm{C}$. (c) The fluorescence distribution from the $90^{\circ}$ detector. Note the population within the vertical gated area: this portion of the spermatozoa is properly oriented to the laser beam. (d) The separation of the $X$ and $Y$ chromosome populations. Each peak population has a sort window represented by vertical lines on the peaks. The area between the two sort windows shows spermatozoa that are mixed ( $X$ and $Y$ ) in various percentages and so they are discarded through the waste. The simultaneously collected $0^{\circ}$ fluorescence distribution of the properly oriented spermatozoa shows a partially resolved bimodal distribution (d). Spermatozoa containing the smaller $Y$ chromosome and thus less total DNA comprise the dimmer (left) population and similarly the larger $\mathrm{X}$-chromosome bearing spermatozoa comprise the brighter population. (e) A schematic of the sorting process, depicting the electrostatic field through which the charged droplets containing $X$ or $Y$ spermatozoa must fall and be deflected to the waiting tubes.

population of sorted spermatozoa will keep and maintain their fertilizing capacity (Johnson et al., 1989). In particular, the current protocol for swine semen has been refined from our original protocol (Johnson, 1991) so that an aliquot of fresh semen containing $15 \times 10^{6}$ spermatozoa are diluted to $1 \mathrm{ml}$ with BTS extender; Hoechst 33342 is added to that suspension at the concentration of $4 \mu \mathrm{g}$ per $15 x$ $10^{6}$ spermatozoa, giving a final concentration of about $7.1 \mu \mathrm{mol} \mathrm{l}^{-1}$. This suspension is then incubated at $32^{\circ} \mathrm{C}$ for approximately $45 \mathrm{~min}$. After the incubation, spermatozoa are uniformly stained so that there is minimal variation in the staining intensity within the $X$ - or $Y$-bearing sperm population, as the most critical part of the separation process depends on uniform staining to maintain resolution of the $X$ and $Y$ sperm populations. The original procedure Uohnson et al., 1989) required incubation at $35^{\circ} \mathrm{C}$ and about $5 \mu \mathrm{g}$ of stain per $10 \times 10^{6}$ spermatozoa and incubation for $1 \mathrm{~h}$. We believe the refined protocol uses the least amount of stain at the lowest temperature to maintain the highest sperm viability after the sorting process. Modifications to the preparation procedures continue as we 
adapt to new instrumentation. For example, spermatozoa and stain concentration is being increased to provide a more concentrated sample for the high speed MoFlo cell sorter now in use in our laboratory (Mo Flo, Cytomation, Inc.; L. Johnson and G.Welch, unpublished).

\section{Collection of the sorted $X$ and $Y$ spermatozon populations}

A critical aspect to maintaining viability of sperm populations that have been put through a cell sorter is to provide an environment that will minimize the dilution that accompanies sperm sorting. The concentration of the stained boar spermatozoa to be sorted is lower $\left(15 \times 10^{6} \mathrm{ml}^{-1}\right)$ than that of the original ejaculates (about $300 \times 10^{6} \mathrm{ml}^{-1}$ ) and even the concentrations used for insemination $\left(25-30 \times 10^{6} \mathrm{ml}^{-1}\right)$. Once the fluorescently stained spermatozoa pass into the flow cell, the stream is surrounded by a sheath fluid of phosphate-buffered saline (PBS) containing $0.1 \%(w / v)$ BSA which contributes to an increasing dilution effect. Dilution is minimized by sorting spermatozoa into a tube containing Test extender containing hens' egg yolk (Johnson et al., 1989). The Test-yolk (20\%) has been the most successful for this purpose (Johnson, 1991). Our procedure uses $50 \mu \mathrm{l}$ of Test-yolk per $0.5 \mathrm{ml}$ microcentrifuge tube and this provides a concentrated environment for spermatozoa to migrate to. Under sorting conditions the fluid volume in the tube increases as sorting progresses. The spermatozoa are falling or being projected into the tube and the motile spermatozoa continue their downward movement into the Test-yolk extender at the bottom of the tube and remain there in a concentrated environment. Once sorting is terminated, spermatozoa may be incubated for $20 \mathrm{~min}$ at room temperature. The resultant supernatant containing immotile spermatozoa is drawn off and the concentrated portion is used for insemination. Centrifugation ( $300 \mathrm{~g}$ for $5-8 \mathrm{~min}$ ) can also be used to concentrate the sorted spermatozoa with minimal effect on sperm motility. Centrifugation is necessary if the sorted sample is to be used for IVF.

All of the sorting process takes place at about $22^{\circ} \mathrm{C}$. The use of the sort collection process described above is most effective for spermatozoa to be used for insemination. We also found that when the spermatozoa are being used for IVF to produce sexed embryos, the percentage of yolk in the Test-yolk can be reduced to less than $5 \%$ so that it will not interfere with fertilization during IVF (Johnson, 1995; Rath et al., 1995). A concentration of $2 \%$ yolk is sufficient to provide a concentrated environment for sorted swine spermatozoa (Rath et al., 1997).

Seminal plasma may also be used as a portion of the collection fluid to minimize the dilution effect. In some recent experiments Maxwell et al. (1996), and Maxwell and Johnson (1997) showed that if Test-yolk (2\%) contained $10 \%$ seminal plasma, the percentage of spermatozoa undergoing the acrosome reaction during or after sorting was reduced. However, additional results using the seminal plasma protocol in combination with IVF in pigs showed that fertilization by sorted spermatozoa could be inhibited when the spermatozoa were collected into a Test-yolk (2\%) medium containing $10 \%$ seminal plasma (W. Maxwell, C. Long and L. Johnson, unpublished). Premature capacitation is clearly a problem with sorted boar spermatozoa, just as it is with frozen boar semen. Much more research on the characterization and composition of the collection fluid remains to be done with or without seminal plasma as a component of the collection fluid.

\section{Rate of production of sexed spermatozon}

Use of cell sorting systems such as the Epics V and 750 (Johnson et al., 1989) and the FacStar equipment (Cran et al., 1995) allows sorting rates of viable $X$ and $Y$ spermatozoa of about $3 \times 10^{5}$ to $4 \times 10^{5}$ spermatozoa $\mathrm{h}^{-1}$. The rate is slow because increased flow rate of the spermatozoa through the system tends to increase the coefficient of variation in the analysis. Spermatozoa must be orientated to the laser beam to differentiate their $X$ and $Y$ spermatozoa DNA content accurately. Only $20-25 \%$ of the spermatozoa that go through the system can be resolved into $X$ - and Y-bearing populations. The lower rate is based on the need to achieve a high purity of the individual sperm populations. Although $100 \%$ purity cannot be achieved in a livestock sperm population with this flow sorting system under normal sorting conditions, $95 \%$ purity can be achieved (Johnson, 1992). In some 
species in which the difference in DNA is greater, such as in the creeping vole (Microtus oregoni; 12.5\%; Johnson and Clarke, 1990) and the Chinchilla Chinchilla laniger (7.5\%; Johnson et al., 1987a) $100 \%$ purity can be achieved. Highest purities attained in flow sorting depend on (i) genetically determined difference in DNA content between the X-and Y-chromosome bearing spermatozoa, (ii) proper configuration and sensitivity of the flow system to allow selection of a properly orientated population of spermatozoa coincident with optimized sort windows, and (iii) uniform and consistent binding of the fluorochrome to the DNA in the sperm head.

\section{Purity of sorted spermatozoa}

The aforementioned factors are critical for achieving high sort purity. If $20 \times 10^{4}$ spermatozoa are used for sorting, sorting for $2 \mathrm{~h}$ will use about $65 \%$ of the spermatozoa prepared. This results in the accumulation of approximately $8 \times 10^{8}$ sorted $X$ spermatozoa and the same number of sorted $Y$ spermatozoa with sort windows set to achieve $80-90 \%$ purity of either $X$ or $Y$ spermatozoa, i.e. about $8 \%$ of all droplets formed are available for sorting. Decreasing the sort window by $15 \%$ results in a $10 \%$ increase in purity $(>90 \%$ ). This can result in about a $20 \%$ reduction in spermatozoa that can be sorted over $2 \mathrm{~h}$. Viability remains unaffected by manipulations of the sort windows. Sperm motility and acrosomal integrity of sorted spermatozoa are slightly lower than those of the original sample.

\section{Laser and stain effects}

Hoechst stained spermatozoa require an excitation source in the ultraviolet (UV) range. Since Hoechst 33342 is the only effective stain for sorting viable intact spermatozoa (Johnson et al., 1989), there is currently no alternative to a UV laser for sorting spermatozoa based on DNA content. The duration of exposure to illumination is determined by the flow velocity of the spermatozoa as it traverses the laser beam (2-3 $\mu \mathrm{s})$. The intensity of laser illumination is determined by the output power of the laser and is readily adjustable. We have investigated the impact of laser power on both the ability to resolve $X$ from $Y$ spermatozoa and their fertilizing capability.

Since spermatozoa are being subjected to UV irradiation during the sorting process, it is useful to reduce laser power to the minimum necessary to obtain resolution between the $X$ and $Y$ peaks (Fig. 2). Much of our initial work was done at about $175 \mathrm{~mW}$ of laser power. More recently we found that resolution could be maintained if the power was reduced to $75-125 \mathrm{~mW}$. At below $75 \mathrm{~mW}$ the loss of resolution became more pronounced. Power reductions affected the resulting purities of the Y sperm population to the greatest extent. Even at low laser power (below $125 \mathrm{~mW}$ ) where the resolution of $X$ and $Y$ spermatozoa appeared diminished, the $X$ sperm population could still be sorted to $90 \%$ purity while $Y$ spermatozoa purity fell to $70 \%$. Fertilization rates in rabbits with spermatozoa sorted using various laser powers showed a more pronounced change at $125 \mathrm{~mW}$. The percentage of unfertilized eggs decreased from $60 \%$ at $200 \mathrm{~mW}$ to $25 \%$ at $125 \mathrm{~mW}$. Similarly, the percentage of 8-16-cell and early morula embryos increased from $30 \%$ at $200 \mathrm{~mW}$ to $70 \%$ at $125 \mathrm{~mW}$ (Johnson et al., 1996).

\section{Potential for Alternative Method of Sexing Semen Based on Surface Protein}

It is well understood that a method for sexing semen on a large scale, at least a scale that could be applied to semen production practice at an AI Centre, would be advantageous. The method most often referred to in this context is that of isolating a protein from the surface of the X- or Y-bearing spermatozoa that is chromosome specific and thus sex specific. The theory goes that if such a marker could be isolated, an antibody could be developed to attach to that X-or Y-bearing population of spermatozoa. The assumption is that the use of affinity chromatography or magnetic beads would provide a large-scale separation process for separating $X$ - from $Y$-bearing spermatozoa and be readily adaptable for AI Centre use. The first step in this process is to identify a surface protein. Boar spermatozoa were sorted for $X$ or $Y$ populations at purities of $90 \%$. Approximately 1000 proteins 

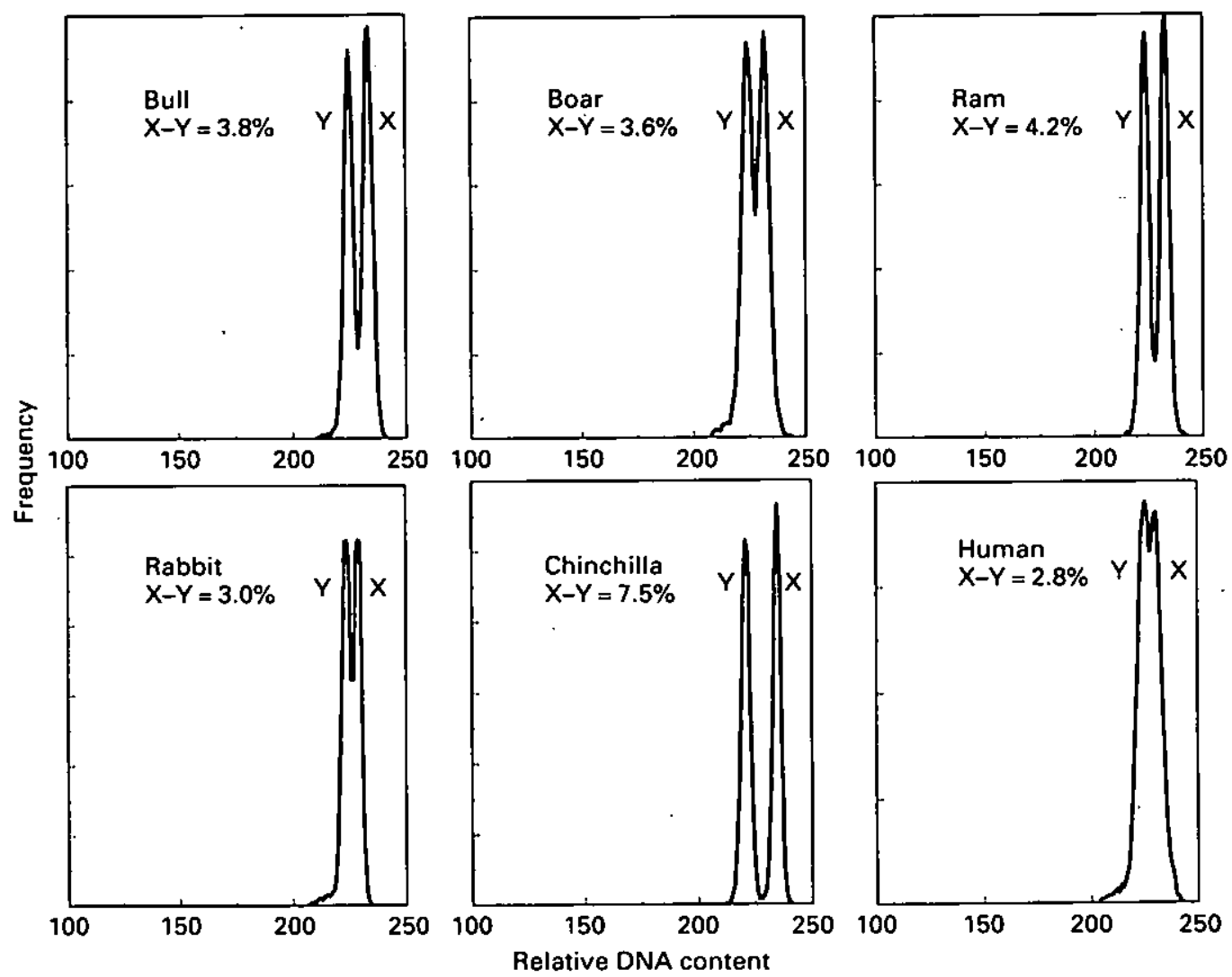

Fig. 2. The relative DNA content of spermatozoa measured by flow cytometry from six different species. DNA content is not comparable between species, as instrument settings dictate histogram position. (These data are also summarized in Johnson et al., 1987a; Johnson 1992; 1994; 1995).

were mapped on the sperm surface. However, no difference could be detected between the proteins isolated from $X$ spermatozoa versus $Y$ spermatozoa. These results would lead to the contention that there are no sex specific proteins on the surface of the spermatozoa (Hendriksen et al., 1996) and that the likelihood of developing a batch process based on an antibody separation is doubtful.'

\section{Embryo Development}

\section{Fertilization and early development in vivo}

The observation that reduced litter size in does and sows inseminated with flow sorted $X$ - or Y-bearing spermatozoa (Johnson et al., 1989; Johnson, 1991) led us to evaluate early embryo development to determine the impact of flow sorted spermatozoa in rabbits. McNutt and Johnson (1996) found an influence of sorting on development of embryos flushed at $42 \mathrm{~h}$ after surgical insemination. Embryos had reached the 8-16-cell stage, whereas embryos from the controls (unstained, unsorted spermatozoa) had already progressed to the early morula stage $(P<0.01)$. The impact seen early in development was carried through pregnancy since there were significantly fewer fetuses from inseminations with flow-sorted spermatozoa than from inseminations with unsorted, unstained, control spermatozoa. Results from cattle (Cran et al., 1993) indicated that most embryos produced from IVF using non-stained, non-sorted spermatozoa produced fully expanded 
blastocysts 7 days after insemination. When stained, sorted spermatozoa were used for IVF, there was a delay of $12 \mathrm{~h}$ in blastocyst development. These studies provided some evidence that when flow sorted spermatozoa are used for fertilization there is a definite impact on the early and subsequent fetal development. Questions are frequently raised about the fertilization process and competence of embryos produced by IVF. To test this hypothesis, we added the Hoechst 33342 stain to fresh boar semen and then inseminated gilts. At two different concentrations of Hoechst 33342, no impact was seen on the developmental competence of the resultant embryos (Garner et al., 1996).

\section{Production of embryos through in vitro fertilization in swine}

The usefulness of the sexing technology in combination with IVF was demonstrated in our laboratory using oocytes matured in vivo and IVF (Rath et al., 1997). Cleavage rates after IVF were $56 \%(n=367)$ and resultant $2-4$-cell embryos were transferred to $24 \mathrm{~h}$ asynchronous $(n=4)$ gilts. Two pregnancies resulted from these initial trials and produced litters of six and four pigs. All embryos transferred were produced from IVF using $X$-sorted spermatozoa and all offspring were female. These were the first pigs born from a combination of the Beltsville Sperm Sexing Technology and IVF in the pig (Table 2). Techniques to improve IVF for swine continue in our laboratories (C. Long, J. Dobrinsky, L. Johnson, unpublished). Once cleavage rates and blastocyst development rates reach the $70 \%$ rate with coincident reductions in polyspermy, the use of IVF/sexed spermatozoa in pigs will be advanced. As mentioned earlier, one factor that needs to be controlled carefully in using sexed spermatozoa is that they are used soon after sorting. This need is based on the premature capacitation that is induced by the sorting process. Recent results with small numbers of liquid stored spermatozoa and artificial insemination in cattle suggest that even at the current sexed sperm production rates, there may be applicability for AI of $1-2 \times 10^{5}$ per insemination (Seidel et al., 1997). It is unlikely that the technology for small numbers of spermatozoa from cattle will be directly applied to swine because of the anatomy of the pig uterus which provides an impediment to small numbers of spermatozoa. This is an area of research that could benefit greatly from more intensive research effort. Indeed, the whole swine artificial insemination industry would benefit from greater research emphasis, since the increasing use of $\mathrm{AI}$ will bring about increasing pressure for greater economy of spermatozoa per insemination dose.

\section{Laboratory Validation of Sperm Sorting Results}

\section{Reanalysis of sorted spermatozoa for DNA}

At some point during a sort or immediately after the completion of a sort to be used for insemination or IVF, a presiliconized BSA-treated tube is placed in position to collect the sorted spermatozoa. No Test-yolk is added to the tube. Approximately $10^{5}$ spermatozoa are sorted into each tube. After the aliquot has been sorted, the spermatozoa are sonicated to remove the tails, and Hoechst 33342 added to maintain staining uniformity and then flow cytometrically reanalysed but not resorted (Johnson et al., 1989). The proportion of X-and Y-bearing spermatozoa are determined based on the DNA difference and histograms are analysed by computer fitting to double gaussian peaks (Fig. 2; Johnson et al., 1987b).

\section{Molecular genetic analysis of single sorted spermatozoa}

A qualitative determination of the presence of the $X$ or $Y$ chromosome in an individual spermatozoan can be made by using the allele-specific polymerase chain reaction (PCR). By analysing several hundred individual spermatozoa a quantitative determination of the proportion of $X$ and $Y$ spermatozoa in a given population can be made (Welch et al., 1995). This method also has application for genetic testing using single cell technologies. Single spermatozoa determined to be $X$ or $\mathrm{Y}$ by flow cytometric analysis were deposited into each well of a 48 well plate. The wells are 
Table 2. Farrowing results from gilts receiving IVM/IVF embryos produced from sexed spermatozoa"

\begin{tabular}{|c|c|c|c|c|}
\hline \multirow[b]{2}{*}{ Treatment } & \multirow{2}{*}{$\begin{array}{l}\text { Litter } \\
\text { size }\end{array}$} & \multicolumn{2}{|c|}{ Offspring } & \multirow{2}{*}{$\begin{array}{c}\text { Predicted } \\
\operatorname{sex}^{6}(\%)\end{array}$} \\
\hline & & Male & Female & \\
\hline Sorted for $X$ & 6 & 0 & 6 & 89 \\
\hline Sorted for $X$ & 4 & 0 & 4 & 89 \\
\hline
\end{tabular}

-Data from Rath et al. (1997).

'By reanalysis of sorted spermatozoa for DNA.

shallow enough to allow microscopic verification of the contents of the wells after single spermatozoa sorting. The highly conserved zinc finger allele which resides on both the $X$ and $Y$ chromosome (ZFx and Zfy) was amplified using nested allele-specific PCR. The wells also serve as the first round amplification vessel for PCR. Specific $X$ and $Y$ products from a second amplification are separated by agarose gel electrophoresis; product bands are identified and counted; and the resulting purities are determined. Results from 100 amplifications of each $X$ and $Y$ sort showed $94 \%$ purity for $X$ and $90 \%$ purity for $Y$ (Welch et al., 1995). These purities are consistent with results obtained by flow cytometric reanalysis of sorted spermatozoa described above.

\section{Embryo sexing by PCR}

Embryos that have been produced from IVF with sorted spermatozoa are frozen by standard methods and stored for later analysis of gender by PCR. After thawing and incubation with proteinase $\mathrm{K}$, the DNA is probed using primers derived from a multiple repeat bovine $\mathrm{Y}$ chromosome specific sequence (Cran et al., 1995). This is an effective way to ensure almost $100 \%$ accuracy in the sex of the embryo.

\section{Fluorescence in-situ hybridization (FISH) for validating proportions of $X$ and $Y$ spermatozon}

Application of FISH to determination of $X$ and $Y$ proportions is fairly straightforward with human spermatozoa because of the availability of commercial microsatellite centromeric DNA probes. Our initial work on humans was validated using FISH (Johnson et al., 1993). Spermatozoa were sorted on to slides, fixed with methanol and acetic acid, exposed to biotinylated DNA probes for $\mathrm{X}$ or $\mathrm{Y}$ chromosomes and incubated; the signals indicating an $\mathrm{X}$ or $\mathrm{Y}^{\prime}$ chromosome were then counted using fluorescence microscopy. This technique is particularly helpful when the difference between $X$ and $Y$ DNA $(<3.0 \%)$ is small and DNA reanalysis by flow cytometry is inconsistent (Johnson et al., 1993).

Recently we have completed a study using FISH and a Y-specific DNA probe developed by T. Kawarasaki for pig spermatozoa. In a comparative study using sorted boar spermatozoa, there was no significant difference between the purities obtained using FISH and those obtained from reanalysing an aliquot of the sorted spermatozoa for DNA (Kawarasaki $e t$ al., 1997).

\section{Birth of Offspring From Sorted $X$ and $Y$ Spermatozoa}

Studies to validate the efficacy of the Beltsville Sperm Sexing Technology were initially conducted in rabbits and swine. The sex ratios of the litters produced from females surgically inseminated with sorted $X$ or $Y$ spermatozoa supported the results of reanalysing aliquots of sorted sperm populations (Johnson et al., 1989; Johnson, 1991). A summary of the results obtained in these studies is presented in Tables 2 and 3 . In the latter study with pigs, spermatozoa were sorted in the usual way into Test-yolk 
Table 3. Progeny produced from intratubal insemination of pig spermatozoa sorted into $\mathrm{X}$ - or Y-chromosome bearing populations

\begin{tabular}{|c|c|c|c|c|c|c|c|}
\hline \multirow[b]{3}{*}{ Treatment } & \multirow{3}{*}{$\begin{array}{c}\text { Number } \\
\text { inseminated }\end{array}$} & \multirow{3}{*}{$\begin{array}{l}\text { Number } \\
\text { farrowed }\end{array}$} & \multirow{3}{*}{$\begin{array}{c}\text { Number } \\
\text { bom }\end{array}$} & \multicolumn{4}{|c|}{ Offspring } \\
\hline & & & & \multicolumn{2}{|c|}{$\begin{array}{c}\text { Actual } \\
(\%)\end{array}$} & \multicolumn{2}{|c|}{$\begin{array}{c}\text { Predicted sex } \\
(\%)\end{array}$} \\
\hline & & & & Female & Male & $x$ & $\mathrm{Y}$ \\
\hline \multicolumn{8}{|l|}{ Early technology } \\
\hline Sorted for $Y$ & 8 & 4 & 37 & 32 & 68 & 23 & 77 \\
\hline Sorted for $X$ & 10 & 5 & 34 & 74 & 26 & 80 & 20 \\
\hline Unsorted/stained & 11 & 5 & 40 & 57 & 43 & 50 & 50 \\
\hline Unsorted/unstained & 7 & 5 & 46 & 48 & 52 & 50 & 50 \\
\hline \multicolumn{8}{|l|}{ Recent technology ${ }^{b}$} \\
\hline Sorted for $Y$ & 1 & 1 & 7 & 14 & 86 & 15 & 85 \\
\hline Sorted for $X$ & 1 & 1 & 8 & 88 & 12 & 89 & 11 \\
\hline
\end{tabular}

-Johnson (1991).

'Rath et al. (1997).

'By reanalysis of sorted spermatozoa for DNA.

(20\%) extender and inseminated via the uterus into the oviduct. In most cases the gilts were synchronized and ovulated ( $42 \mathrm{~h}$ after hCG administration) about $4 \mathrm{~h}$ before surgical intervention. The results demonstrate a significant shift in the sex ratio of the litters born. However, the proportion of one sex or the other ( $68 \%$ for males and $74 \%$ for females) is lower than that achieved with rabbits. One of the reasons for this is that boar spermatozoa do not orientate as efficiently as rabbit spermatozoa. With current refinements and technology, the shift in the sex ratio for swine has improved to $85-90 \%$ using standard conditions. No effort has been made to inseminate gilts with regular AI since the number of spermatozoa required is prohibitive with respect to sorted spermatozoa. As mentioned earlier, sorted spermatozoa have been used for IVF and two litters of pigs have been produced; all were female (Table 2). This work (Rath et al., 1997) demonstrated the feasibility of using IVF and embryo transfer in conjunction with the sexing technology.

\section{Conclusions}

Preselection of sex of progeny in livestock has been validated on the basis of live births, on the basis of reanalysis of sorted spermatozoa for DNA content on the basis of PCR and on the basis of determining the sex of the resulting embryos. The cell sorting method to separate $X$ - and $Y$ - bearing spermatozoa can be used in conjunction with IVF in swine and in cattle for the production of sexed embryos to be transferred to eligible recipients for the duration of gestation. This semi-practical sexing method, although impractical for some production systems, could be used to provide a more flexible progeny producing option in many livestock operations. Flow cytometric sorting of spermatozoa for reproductive purposes requires significant commitment in expertise and resources in order to apply the systems commercially or in research situations. Research continues to improve the efficiency of sorting more spermatozoa per unit time. Current research involving the removal of dead spermatozoa from the sorting process through the addition of the dead membrane permeant propidium iodide as well as other dyes in combination with Hoechst 33342 can result in a more efficiently sorted population (Johnson et al., 1994). High speed sorting (MoFlo, Cytomation, Inc.) as is now used in our laboratory will increase the throughput of sorted spermatozoa per unit time. Improvements in the technology will no doubt lead to much greater use of sexed spermatozoa, depending on the species involved. Solving the problems associated with inseminating small numbers of sexed spermatozoa in pigs would be a great benefit to AI and swine production. 


\section{References}

Amann RP and Seidel GE (Eds) (1982) Prospects for Sexing Mammalian Spermatozon Colorado University Press, Boulder

Barlow $P$ and Vosa CG (1970) The $Y$ chromosome in human spermatozoa Nature 226 961-962

Catt SL, Catt JW, Gomez MC, Maxwell WMC and Evans G (1996) Birth of a male lamb derived from an in vitro matured oocyte fertilised by intracytoplasmic injection of a single presumptive male spermatozoa Veterinary Record 139 494-495

Cran DG, Johnson LA, Miller NG, Cochrane D and Poige C (1993) Production of bovine calves following separation of $X$ - and Y-chromosome bearing spermatozoa and in vitro fertilisation Veterinary Record 132 40-41

Cran DG, Johnson LA and Polge C (1995) Sex preselection in cattle: a field trial Veterinary Record 136 495-496

Cui K (1997) Size differences between human $X$ and $Y$ spermatozoa and prefertilization diagnosis Moircular Human Reproduction 3 61-87

Dean PN, Pinkel D and Mendelsohn ML, (1978) Hydrodynamic orientation of spermatozoa heads for fow cytometry Biophysics Journal 23 7-13

Ericsson RJ, Langerrin CN and Nishirro $M$ (1973) Isolation of fraction-rich in human $Y$ spermatozoa Nature 246 421-424

Garner DL, Dobrinsky JR, Welch GR and Johnson LA (1996) Porcine spermatozoa viability, oocyte fertilization and embryo development after staining spermatozoa with SYBR-14 Theriogenology 45 1103-1113

Gledhill BL (1988) Gender preselection: historical, technical, and ethical perspectives Seminars in Reprodtictive Endocrinology 6 385-395

Gledhill BL, Lake S, Steinmetz LL, Gray JW, Crawford JR, Dean PN and Van Dilla MA (1976) Flow microfluorometric analysis of spermatozoa DNA content: effect of cell shape on the fluorescence distribution Journal of Cell Physiology 87 367-376

Hendriksen PJM, Tieman $M$, Van Der Lende T and Johnson LA (1993) Binding of anti-H.Y monoclonal antibodies to separated $X$ and $Y$ chromosome bearing porcine spermatozoa Molectular Reproduction and Development 5 189-196

Hendriksen PJM, Welch GR, Grootegoed JA, Van Der Lende T and Johnson LA (1996) Comparison of detergent-solubilized membrane and soluble proteins from flow cytometrically sorted $X$ - and Y-chromosome bearing porcine spermatozoa by high resolution 2-D electrophoresis Molecular Reproduction and Development 45 342-350

Johnson LA (1988) Flow cytometric determination of spermatozoa sex ratio in semen purportedly enriched for $X$ or $Y$ bearing spermatozoa Theriogenology 29265

Johnson LA (1991) Sex preselection in swine: altered sex ratios in offspring following surgical insemination of flow sorted $X$ - and Y-bearing spermatozoa Reproduction in Domeslic Animals 26 309-314

Johnson LA (1992) Cender preselection in domestic animals using flow cytometrically sorted spermatozoa Journal of Animal Science 70 (Supplement 2) 8-18

Johnson LA (1994) Isolation of X-and Y-bearing spermatozoa for sex preselection In Oxford Reviews of Reproductive Biology Ed. HH Charlton, pp 303-3226. Oxford University Press, Oxford

Johnson LA (1995) Sex preselection by flow cytometric separation of $X$ and $Y$ chromosome-bearing spermatozoa based on DNA difference: a review Reproduction Fertility and Developoment 7 893-903

Johnson LA and Clarke RN (1988) Flow sorting of $X$ and $Y$ chromosome-bearing mammalian spermatozoa: activation and pronuclear development of sorted bull, boar and ram spermatozoa microinjected into hamster oocytes Gamete Research 21 335-343

Johnson LA and Clarke RN (1990) Spermatozoa DNA and sex chromosome differences between two geographical populations of the creeping vole, Microtus oregoni. Molecular Reproduction and Development 27 159-162

Johnson LA and Pinkel D (1986) Modification of a laser-based flow cytometer for high resolution DNA analysis of mammalian spermatozoa Cytontetry 7 268-273

Johnson LA, Flook JP, Look MV and Pinkel D (1987a) Flow sorting of $X$ and $Y$ chromosome-bearing spermatozoa into two populations Gamete Research 16 1-9

Johnson LA, Flook JP and Look MV (1987b) Flow cytometry of X and $Y$ chromosome-bearing spermatozoa for DNA using an improved preparation method and staining with Hoechst 33342 Gamete Research 17 203-212

Johnson LA, Flook J P and Hawk HW (1989) Sex preselection in rabbits: live births from $X$ and $Y$ spermatozoa separated by DNA and cell sorting Biology of Reproduction 41 199-203

Johnson L,A, Welch GR, Keyvanfar K, Dorfmann A, Fugger EF and Schulman JD (1993) Gender preselection in humans? Flow cytometric separation of $X$ and $Y$ spermatozoa for the prevention of X-linked diseases Human Reproduction 8 1733-1739

Johnson LA, Welch GR and Garner DL (1994) Improved flow sorting resolution of $X$ and $Y$ chromosomes bearing viable spermatozoa separated using dual staining and dead cell gating Cytometry 17 (Supplement 7) 83

Johnson LA, Cran DG, Welch GR and Polge C (1996) Gender preselection in mammals In Beltsville Symposium $X X$ Biotechnology's Role in the Genetic Inprovement of Farm Animals Ed. RH Miller, VG Pursel and HD Norman. PP 151-164 American Society of Animal Science, Savoy, IL

Kawarasaki T, Welch GR, Long CR, Yoshida $M$ and Johnson LA (1997) Rapid estimation of flow sorted X- and Ychromosome bearing porcine spermatozoa by fluorescence in situ hybridization Molecular Reproduclion and Development (in press)

Kiddy CA and Hats HD (Eds) (1971) Sex ratio at birth - prospects for control Journal of Animal Science Symposium - Supplement American Society for Animal Science Champaign, IL

McNult TL and Johnson LA (1996) Flow cytometric sorting of spermatozoa: influence on fertilization and embryo/fetal development in the rabbit Molecular Reproduction and Development 43 261-267

Maxwell WMC and Johnson LA (1997) Chlortetracycline analysis of boar spermatozoa after incubation, flow cytometric sorting, cooling, or cryopreservation Molecular Reproduction and Development $46408-418$

Maxwell WMC, Welch GR and Johnson LA (1996) Viability and membrane integrity of spermatozoa after dilution and flow cytometric sorting in the presence or absence of seminal plasma Rcproduction Fertility and Development 8 $1165-1168$

Moruzzi Jf (1979) Selecting a mammalian species for the separation of X-and Y-chromosome bearing spermatozoa Journal of Reproduction and Fertility 57 319-323

Pinke] D, Gledhill BL, Van Dilla MA, Stephenson D and 
Watchmaker G (1982) High resolution DNA measurements of mammalian spermatozoa Cytometry 3 1-9

Rath D, Johnson LA, WeJch GR and Niemann H (1995) Successful gamete intrafallopian transfer (GIFT) in the porcine Theriogenology 41 1173-1179

Rath D, Johnson LA, Dobrinsky JR and Welch GR (1997) Production of piglets presclected for sex following in vitro fertilization with $X$ and $Y$ chromosome-bearing spermatozoa sorted by flow cytometry Theriogenology 47 795-800
Seidel GE, Allen CH, Johnson LA, Holland MD, Brink Z, Welch GR, Graham JK and Cattell MB (1997) Insemination of heifers with very low numbers of nonfrozen spermatozoa and sexed spermatozoa Theriogenology (in press)

Welch GR, Waldbieser GC, Wall RJ and Johnson LA (1995) Flow cytometric spermatozoa sorting and PCR to confirm separation of $X$ - and $Y$-chromosome bearing bovine spermatozoa Animal Biotechnology 6 131-139 